\title{
Forecast of Korea Defense Expenditures based on Time Series Models
}

\author{
Kyung Ok Park ${ }^{a}$, Hye-Young Jung ${ }^{1, b}$ \\ ${ }^{a}$ Department of Mathematics, Yonsei University, Korea \\ ${ }^{b}$ Department of Statistics, Seoul National University, Korea
}

\begin{abstract}
This study proposes a mathematical model that can forecast national defense expenditures. The ongoing European debt crisis weighs heavily on markets; consequently, government spending in many countries will be constrained. However, a forecasting model to predict military spending is acutely needed for South Korea because security threats still exist and the estimation of military spending at a reasonable level is closely related to economic growth. This study establishes two models: an Auto-Regressive Moving Average model (ARIMA) based on past military expenditures and Transfer Function model with the Gross Domestic Product (GDP), exchange rate and consumer price index as input time series. The proposed models use defense spending data as of 2012 to create defense expenditure forecasts up to 2025.
\end{abstract}

Keywords: Defense expenditures, GDP, exchange rate, Consumer price index, ARIMA model, Transfer Function model.

\section{Introduction}

Military expenditures include any spending for the purpose of national security; consequently, they are a necessary expense to generate public goods of 'security' (Baek et al., 2002). Spending is necessary because it protects people and their property and because national security is related to economic growth that it demonstrates a nation' willingness to protect national interests. This is particularly relevant in South Korea, a divided nation with an ongoing military confrontation between South and North. The development of a forecasting model to predict defense expenditures is salient due to internal and external security factors such as the transfer of wartime operational control. High spending on national defense is considered to weakens trade competitiveness and disperses investment with a negative influence on economic growth (Mintz and Huang, 1990; Rothschild, 1973); however, Korea has achieved a high rate of economic growth while maintaining high national defense spending (Chan and Mintz, 2002). In this case, the threat of military confrontation between the two Koreas means that a strong commitment to defending national security plays a critical role to boost the South Korean economy. The correlation between Korea's spending on national defense and its economic growth rate supports the hypothesis of Aizenman and Glick (2006), which claims that defense expenditures in the presence of threats have a positive impact on economic growth despite the direct negative impact of military expenditures and security threats on economic growth (Chan and Mintz, 2002).

Factors that influence defense expenditures are largely categorized into economic conditions such as fiscal capability and national security threats. A country's existence would be at risk if defense

\footnotetext{
${ }^{1}$ Corresponding author: Department of Statistics, Seoul National University, Seoul 151-747, Korea.

E-mail: hyjunglove@snu.ac.kr
}

Published 31 January 2015 / journal homepage: http://csam.or.kr

(c) 2015 The Korean Statistical Society, and Korean International Statistical Society. All rights reserved. 
spending were too low in the presence of security threats. Excessive spending on defense disproportionate to general economic circumstances would hamper economic growth. Economic conditions have a stronger impact on Korean military expenditures than any other factor because the national policy direction focuses on the growth of the economy (Korean Defense Ministry, 2006). Other issues (such as security threats, the modernization of military facilities, and internal and external issues in response to the transfer of wartime operational control) must also be considered when estimating Korea's defense expenditures.

An appropriate forecast defense spending model is important if the ongoing European sovereigndebt crisis continues to make it impossible for the Korean government to increase spending. Current studies have focused on choosing variables that affect the estimation of defense expenditures and how to allocate them; however, economic conditions matter most when forecasting a country's military expenditures. This study suggests using an ARIMA model (Anderson, 1971; Box and Jenkins, 1976) based on past defense expenditures and a Transfer Function model (William, 1996) with GDP, exchange rate and consumer price index as input variables to generate an appropriate military spending forecasting model. Based on the estimates obtained from these models and other variables that affect the forecast of military spending, security threats and economic conditions, it would be rational for economic and military experts to revise forecasting methods to produce more reasonable forecasts.

This paper sees a sense of urgency to develop a mathematical model based on time series data of past defense expenditures. This paper determines an appropriate forecast model that is mathematically feasible and provides basic data to estimate defense expenditures. This study utilizes and analyzes military spending data from 1970 to 2012 published by the Ministry of National Defense, the Population Projection for Korea released by Statistics Korea, GDP, exchange rate and consumer price index released by the Bank of Korea.

\section{The Forecasts of Korea Defense Expenditures based on the Univariate Time Series Model}

The defense expenditure per person $\left(z_{t}\right)$ is a sequence of observations taken over time and it is needed to investigate the stationary, trend, periodicity and autocorrelation before analyzing the defense expenditure data.

The ARIMA model developed by Box and Jenkins (1976) assumes that the time series is stationary; therefore, raw data is transformed to defense expenditure per person to obtain stationary time series that has a constant mean and a constant variance. It gradually increases over time and shows that variance stabilization is needed through data transformation (Figure 1). We used square root transformation in order to obtain data with constant variance.

Table 1 shows that since the $p$-values from a unit root test of $\sqrt{z_{t}}$ are not significant, unit root exists. If the presence of a unit root is not rejected, then one should apply the difference operator to the series. After differencing, we consider ARIMA model. Figure 2 confirms that the first differenced square root transformed data is stationary, with a constant mean and a constant variance. The three types of parameters in the $\operatorname{ARIMA}(p, d, q)$ model are: the autoregressive parameter " $p$ ", the number of differencing passes " $d$ ", and the moving average parameter " $q$ ". In general, the parameters $\mathrm{p}$ and $q$ in the model can be identified based on the shape of the autocorrelation function (ACF) and partial auto correlation function (PACF). Since ACF and PACF show that there is drastic decreasing after time lag 1 from Figure 3, we start with an $\operatorname{ARIMA}(1,1,1)$ model, then the $\operatorname{ARIMA}(1,1,0)$ model, finally, the $\operatorname{ARIMA}(0,1,1)$ model. Table 2 compares two models having significant estimates using Akaike Information Criterion(AIC) and Schwartz Bayesian Criterion(SBC). In Table 2, AIC and SBC 

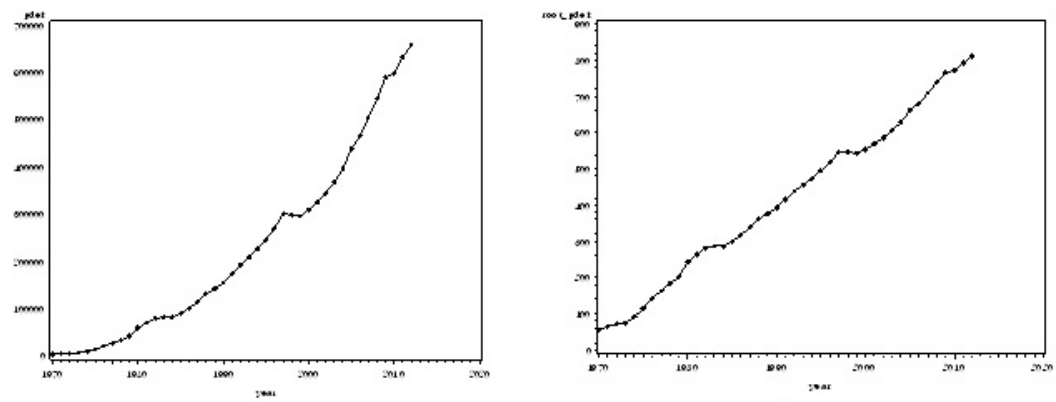

Figure 1: Time series plot of $z_{t}$ (left) and $\sqrt{z_{t}}($ right $)$

Table 1: Unit root test of $\sqrt{z_{t}}$

\begin{tabular}{ccccc}
\hline \hline Model & White noise & AR(1) & AR(2) & AR(3) \\
\hline$p$-value & 0.994 & 0.967 & 0.959 & 0.945 \\
\hline \hline
\end{tabular}

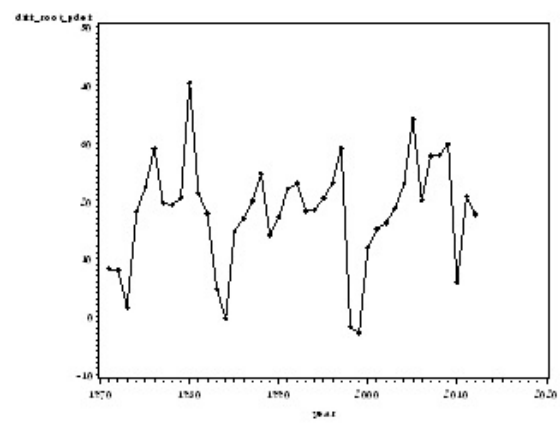

Figure 2: Time series plot of $\nabla \sqrt{z_{t}}$

Table 2: AIC and SBC for the suggested models

\begin{tabular}{ccc}
\hline \hline Model & AIC & SBC \\
\hline ARIMA $(1,1,0)$ & 309.26 & 312.78 \\
ARIMA $(0,1,1)$ & 309.89 & 313.41 \\
\hline \hline
\end{tabular}

choose the $\operatorname{ARIMA}(1,1,0)$ model as the best identified model.

Table 3 shows estimates and standard errors of parameter from the identified model. The estimates are significant with $p$-values less than 0.05 . After we verify the residuals are not autocorrelated and are white noise, the identified ARIMA $(1,1,0)$ model is considered to be a proper model for forecasting.

Table 4 shows Portmanteau's chi-square test to check the assumption that the residuals are a random series such as white noise. We can conclude that the ARIMA $(1,1,0)$ model is considered to be proper because the $p$-value is adequate based on the autocorrelation diagnosis proposed in Table 4 .

Following is the final $\operatorname{ARIMA}(1,1,0)$ model for $\sqrt{z_{t}}$, the square root transformed defense expenditure per person.

$$
\sqrt{z_{t}}=11.05+\sqrt{z_{t-1}}+0.38\left(\sqrt{z_{t-1}}-\sqrt{z_{t-2}}\right)+\epsilon_{t} .
$$

The correlation coefficient between the square root transformed defense expenditure per person of this 


\begin{tabular}{|c|c|c|c|c|c|c|}
\hline Lag. & Correlation. & 987654321. & 123456789 & Correlation. & 987654321 & 123456789 \\
\hline 1. & 0.38250 & $\therefore$ & 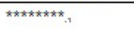 & 0.38250 & $\therefore$ & $\star \star \star \star \star \star \star \star \star \star \star \star *, .1$ \\
\hline 2. & 0.06649 & $\therefore$ & * $\therefore$ & -0.09350 & $x * \star$ & $\therefore$ \\
\hline 3. & -0.15721 & . $\quad * \pi \star \ldots$ & $\therefore$ & -0.17683 & $\cdot * \pi \approx *$. & $\therefore$ \\
\hline 4. & -0.10496 & **. & $\therefore$ & 0.03291. & $\therefore$ & * $\therefore$ \\
\hline 5. & -0.14557 & $* \pi *$ & $\therefore$ & -0.12915 & $\cdot * \pi *$ & $\therefore$ \\
\hline 6. & -0.18425 & 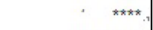 & $\therefore$ & -0.14324 & $\cdot * * *$ & $\therefore$ \\
\hline 7. & -0.34117 & 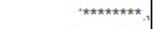 & $\therefore$ & -0.28445 & $* \star * \pi * * \ldots$ & $\therefore$ \\
\hline 8. & -0.21966 & $\cdot \quad * \star \star *$ & $\therefore$ & -0.05141 & $x^{*}$ & $\therefore$ \\
\hline 9. & -0.19444 & $* \star \star$ & $\therefore$ & -0.20751 & $\cdot * \star \star \star \star *$. & $\therefore$ \\
\hline 10. & -0.03438 & $\star$ & $\therefore$ & -0.07445 & . $\quad *$ & $\therefore$ \\
\hline 11. & 0.14181 & 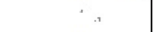 & $\approx * \star \quad \therefore$ & 0.09150 & $\therefore$ & ** $\therefore$ \\
\hline 12. & 0.21618 & $\cdot$ & 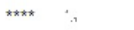 & -0.01221 & $\therefore$ & $\therefore$ \\
\hline 13. & 0.01035 & $\therefore$ & $\therefore$ & -0.26150 & $x_{* \star \star \star \star * * .}$ & $\therefore$ \\
\hline 14. & 0.10259. & $\therefore$ & $\therefore$ & 0.09747 & $\therefore$ & \#夫 $\therefore$ \\
\hline 15. & 0.18918. & $\because$ & $\star \star \star \star \star \therefore$ & 0.12738 & $\therefore$ & $\star \star \star \star ;$ \\
\hline 16. & 0.18776 & $\therefore$ & $\star \star \star \star \star \quad \therefore$ & -0.08453 & $x^{* *}$ & $\therefore$ \\
\hline $17 .+$ & 0.15648 & $\because$ & $\star \star \star * \quad \therefore$ & 0.15724 & $\therefore$ & $\star \star \star * \cdots$ \\
\hline 18. & -0.12013 & . & $\therefore$ & -0.15854 & $\cdot * \star * *$ & $\therefore$ \\
\hline 19. & -0.17844 & . $\quad * \pi * * *$ & $\therefore$ & -0.01564 & $\because$ & $\therefore$ \\
\hline 20. & -0.09200 & . & $\therefore$ & 0.07088 & $\because$ & $\therefore$ \\
\hline
\end{tabular}

Figure 3: $A C F\left(\right.$ left) and $P A C F($ right $)$ of $\nabla \sqrt{z}_{t}$

Table 3: The parameter estimation of $\operatorname{ARIMA}(1,1,0)$ model

\begin{tabular}{cccccc}
\hline \hline Parameter & Estimate & Standard Error & $t$-value & $P$-value & Lag \\
\hline MU & 17.82 & 2.10 & 8.49 & $<.0001$ & 0 \\
AR 1,1 & 0.38 & 0.14 & 2.68 & 0.01 & 1 \\
\hline \hline
\end{tabular}

Table 4: The Portmanteau's chi-square test of residuals

\begin{tabular}{|c|c|c|c|c|c|c|c|c|c|}
\hline To Lag & $\chi^{2}$ & DF & $P$ & & & ocorrelat & & & \\
\hline 6 & 2.02 & 5 & 0.85 & 0.034 & -0.017 & -0.187 & -0.005 & -0.065 & -0.032 \\
\hline 12 & 11.23 & 11 & 0.42 & -0.276 & -0.059 & -0.144 & -0.026 & 0.108 & 0.219 \\
\hline 18 & 18.77 & 17 & 0.34 & -0.135 & 0.054 & 0.133 & 0.096 & 0.181 & -0.155 \\
\hline 24 & 25.53 & 23 & 0.32 & -0.145 & -0.004 & 0.015 & -0.131 & -0.095 & -0.159 \\
\hline
\end{tabular}

year and the square root transformed defense expenditure per person of last year is 0.38 .

\section{The Forecasts of Korea Defense Expenditures using the Transfer Function}

Defense expenditure is affected by national financial affairs. Korea's policy direction focuses on growing the economy; consequently, economic conditions have a stronger impact on its military expenditures than other factors (Aizenman and Glick, 2006). Therefore, we propose a Transfer Function model that has $\operatorname{GDP}\left(g_{t}\right)$, exchange rate $\left(c_{t}\right)$ and consumer price index $\left(x_{t}\right)$ as input time series variables, and defense expenditure per person $\left(z_{t}\right)$ as the output time series variable. Transfer function model is a statistical model describing the relationship between an output variable and one or more input variables. If input series $X_{1, t}, \ldots, X_{k, t}$ can be represented as an $\operatorname{ARIMA}(p, d, q)$ model, then we obtain the transfer function model

$$
Y_{t}=\sum_{i=1}^{k} \frac{w_{i, s}(B)}{\delta_{i, r}(B)} B^{b} X_{i, t}+N_{t},
$$

where $N_{t}$ is a zero-mean stationary process, uncorrelated with $\left\{X_{i, t}, i=1, \ldots, k\right\}, w_{i, s}(B)=w_{i, 0}-$ $w_{i, 1} B-\cdots-w_{i, s} B^{s}$ and $\delta_{i, r}(B)=1-\delta_{i, 1} B-\cdots-\delta_{i, r} B^{r}$.

The parameter $b$ is time lag of input time series having first effects on output time series, $s$ is time lag enduring affects of output time series at time $t$ from input time series at time $t-b$ and $r$ is the 

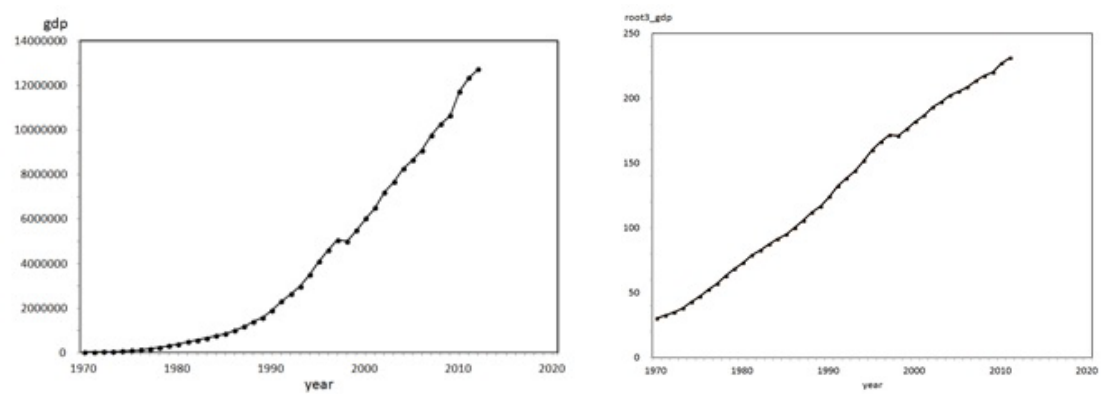

Figure 4: Time series plot of $\operatorname{GDP}(K R W)($ left $)$ and Time series plot of the cube root transformed $G D P(K R W)($ right $)$

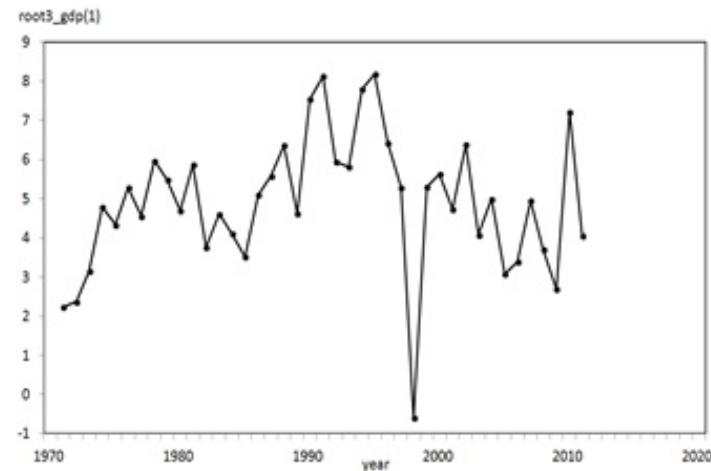

Figure 5: Time series plot of the first differenced cube root transformed GDP $\nabla \sqrt[3]{g_{t}}$

parameter representing the relation between after input time series at time $t-b-s$ and output time series at time $t$. It predicts future values of a time series based on past time series values and on the values of one or more time series related to the time series to be predicted. Our purpose in using the Transfer Function model is to improve the one-variable time series model by analyzing the dynamic relation between input time series data and output time series data. Before setting a transfer function model, the input-output data are first transformed to stationary data, and the $\operatorname{ARIMA}(p, d, q)$ model for input data and output data are found through the prewhitening procedure which changes the input (output) data into white noise beforehand. In order to apply the prewhitening procedure, it is first necessary to investigate the time series plot of GDP. The increase gradually becomes larger as time goes by in Figure 4 (left), so the variance can be stabilized by a cube root transformation of data. The cube root transformed GDP has a trend factor from Figure 4 (right), and it can be removed from first order differencing.

Figure 5 verifies that the data is transformed into an approximately stationary time series which has a constant mean. We choose $\operatorname{ARIMA}(1,1,1)$ as the fitted model since the ACF and PACF for first order differencing GDP after cube root transforming show a spike at time lag 1 in Figure 6 . All estimates in Table 5 are significant, and because $p$-value of Portmanteau's chi-square test is large enough so that the hypothesis "The autocorrelation is equal to zero" cannot be rejected in every time lag as a results of autocorrelation diagnosis of residuals from Table 6.

We can verify that the ARIMA $(1,1,1)$ model of the cube root transformed GDP is suitable since 


\begin{tabular}{|c|c|c|c|c|c|c|}
\hline Lag., & Correlation. & 987654321. & 123456789 & Correlation. & 987654321 & 123456789 \\
\hline 1. & 0.28612 & 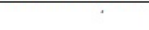 & $* \star \star \star \star \star \star \star * .$. & 0.28612 & $\therefore$ & 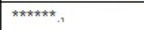 \\
\hline 2. & 0.15438. & & $* * \approx$ & 0.07898 & . & \\
\hline 3. & 0.10258 & & 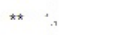 & 0.04307 & & * $\therefore$ \\
\hline 4. & -0.02628 & . $\quad \star_{0}$ & $\therefore$ & -0.08315 & . $* *$ & $\therefore$ \\
\hline 5. & 0.08315 & . & $\approx * \therefore$ & 0.10655 & . . & ** $\therefore$ \\
\hline 6. & -0.04993 & $\cdot \quad *$ & $\therefore$ & -0.10065 & . $* \star *$ & $\therefore$ \\
\hline 7. & -0.07912 & $\cdot \quad * \star *$, & $\therefore$ & -0.05545 & $\cdot \quad *$ & $\therefore$ \\
\hline 8. & -0.02533 & $\cdot \quad *$ & $\therefore$ & 0.00828 & & $\therefore$ \\
\hline 9. & -0.04810 & . $\quad$ * & $\therefore$ & -0.00683 & . & $\therefore$ \\
\hline 10. & -0.07255 & $\cdot \quad$ * & $\therefore$ & -0.07418 & $\cdot \quad *$ & $\therefore$ \\
\hline 11. & 0.01732 & & $\therefore$ & 0.07363 & . & * $\therefore$ \\
\hline 12. & -0.12793 & $\cdot \quad * * * x$, & $\therefore$ & -0.14308 & $\cdot * \star \star x$ & $\therefore$ \\
\hline 13. & 0.05254 & . . & * $\therefore$ & 0.13356 & $\cdot$. & 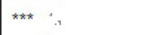 \\
\hline 14. & -0.03512 & $\cdot \quad *$ & $\therefore$ & -0.09452 & $\cdot * x$ & $\therefore$ \\
\hline 15. & -0.11071 & $\cdot \quad * \star \star x$ & $\therefore$ & -0.06336 & . * & $\therefore$ \\
\hline 16. & 0.00912 & . & $\because$ & 0.01720 & & $\therefore$ \\
\hline 17. & -0.18228 & $\cdot x * \pi *$ & $\therefore$ & -0.15238 & $\cdot * \star * x$. & $\therefore$ \\
\hline 18. & -0.18295 & $\cdot \pi \star \star \star \star *$ & $\therefore$ & -0.14482 & $\cdot * \star \star *$ & $\therefore$ \\
\hline 19. & -0.16884 & $\cdot \quad * * \star *$. & $\therefore$ & -0.07287 & . $\quad *$ & $\therefore$ \\
\hline 20. & -0.14778 & $\cdot \quad * \star \star \star *$, & $\therefore$ & -0.02547 & $\cdot \quad *$ & $\therefore$ \\
\hline
\end{tabular}

Figure 6: ACF and PACF of $\nabla \sqrt[3]{g_{t}}$

Table 5: The parameter estimation of $\operatorname{ARIMA}(1,1,1)$ model

\begin{tabular}{cccccc}
\hline \hline Parameter & Estimate & Standard Error & $t$-value & $P$-value & Lag \\
\hline MA1,1 & 0.672 & 0.130 & 5.18 & $<.0001$ & 1 \\
AR1,1 & 0.990 & 0.018 & 55.24 & $<.0001$ & 1 \\
\hline \hline
\end{tabular}

Table 6: The Portmanteau's chi-square test of residuals

\begin{tabular}{|c|c|c|c|c|c|c|c|c|c|}
\hline To Lag & $x^{2}$ & DF & $P$ & \multicolumn{6}{|c|}{ Autocorrelations } \\
\hline 6 & 1.52 & 4 & 0.824 & 0.084 & -0.056 & 0.068 & -0.083 & 0.093 & -0.035 \\
\hline 12 & 3.04 & 10 & 0.981 & -0.007 & 0.013 & -0.004 & -0.068 & 0.042 & -0.136 \\
\hline 18 & 11.24 & 16 & 0.795 & 0.118 & 0.191 & 0.071 & 0.236 & -0.050 & -0.074 \\
\hline 24 & 13.13 & 22 & 0.930 & 0.047 & 0.021 & -0.031 & -0.103 & 0.010 & -0.076 \\
\hline
\end{tabular}

the ACF and PACF of residuals are all included in twice of standard error.

Figure 7 shows that the exchange rate $\left(c_{t}\right)$ and consumer price index $\left(x_{t}\right)$ have to be transformed into the stationary time series by an appropriate transformation. The variance of $c_{t}$ is stabilized by root transformation and then we consider the difference in the transformed data $\sqrt{c}_{t}$. By inspecting the ACF and PACF of $\nabla \sqrt{c_{t}}$, we choose the white noise as the fitted model. Figure 7(right) shows that since the increase gradually becomes larger as time goes by, we can eliminate the trend by first order differencing; consequently, $\nabla x_{t}$ is transformed into an approximately stationary time series. By inspecting the ACF and PACF, we choose the $\operatorname{ARIMA}(1,1,1)$ model as the fitted model of $\nabla x_{t}$.

The parameters $(b, s, r)$ of the Transfer Function model should be decided through cross covariance and cross correlation after fitting the same model to the square root of defense expenditures per person which is the output time series data. First, we consider GDP $\left(g_{t}\right)$. The cross correlation between $\nabla \sqrt{z_{t}}$ and $\nabla \sqrt[3]{g_{t}}$ can be decided to be 0 in negative time lag, which means it is reasonable to use the first order differencing GDP data after square root transforming as the input time series variable of our Transfer Function model. We determine that the parameters $(b, s, r)$ are $(1,0,0)$ or $(1,0,1)$ since the cross correlations between $\nabla \sqrt{z_{t}}$ and $\nabla \sqrt[3]{g_{t}}$ are significant at time lag 1 and not significant after time lag 1 and display a cutoff. Second, we consider exchange rate $\left(c_{t}\right)$. The cross correlations between $\nabla \sqrt{z}_{t}$ and $\nabla \sqrt{c_{t}}$ are significant at time lag 1 and 2 and cut off after lag 2. Thus, we determine that the parameters $(b, s, r)$ are $(1,1,0)$ or $(1,1,1)$. Finally, we consider consumer price index $\left(x_{t}\right)$. By inspecting the cross correlations between $\nabla \sqrt{z}_{t}$ and $\nabla x_{t}$, since cross correlations are not significant 

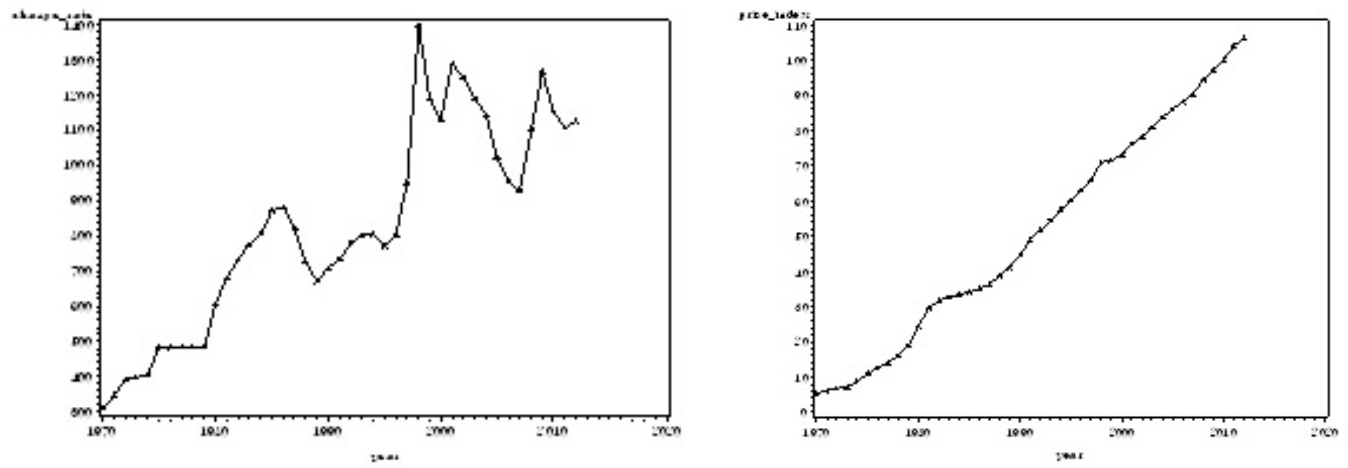

Figure 7: Time series plot of the exchange rate $c_{t}$ (left) and consumer price index $x_{t}$ (right)

Table 7: The maximum likelihood estimation for final model

\begin{tabular}{cccccc}
\hline \hline Input variable & TF model & estimate & Standard Error & $t$-value & $P$-value \\
\hline$\nabla \sqrt[3]{g_{t}}$ & $(1,0,0)$ & 2.34 & 0.638 & 3.67 & 0.0002 \\
$\nabla x_{t}$ & $(0,0,0)$ & 2.54 & 1.209 & 2.10 & 0.0360 \\
\hline \hline
\end{tabular}

Table 8: The Portmanteau's chi-square test of residuals

\begin{tabular}{|c|c|c|c|c|c|c|c|c|c|}
\hline To Lag & $\chi^{2}$ & $\mathrm{DF}$ & $P$ & \multicolumn{6}{|c|}{ Autocorrelations } \\
\hline 6 & 6.54 & 6 & 0.366 & 0.229 & 0.267 & 0.075 & -0.022 & -0.003 & -0.119 \\
\hline 12 & 9.67 & 12 & 0.645 & -0.149 & -0.077 & -0.104 & -0.021 & -0.124 & 0.051 \\
\hline 18 & 14.90 & 18 & 0.669 & -0.161 & -0.082 & -0.096 & -0.096 & 0.044 & -0.151 \\
\hline 24 & 24.09 & 24 & 0.456 & 0.073 & -0.037 & -0.039 & -0.162 & -0.174 & -0.174 \\
\hline
\end{tabular}

and cut off after lag 0 , we decide that the parameters $(b, s, r)$ are $(0,0,0)$ or $(0,0,1)$.

We must search the most satisfactory Transfer Function model with significant independent variables and parameters using parameters of the Transfer Function model to the input variables $\nabla \sqrt[3]{g_{t}}$, $\nabla \sqrt{c_{t}}$ and $\nabla x_{t}$. We can test eight models using the parameters identified above. From results of maximum likelihood estimation to eight models, we choose significant transfer function models $\nabla \sqrt[3]{g_{t}}(1,0,0)$ and $\nabla x_{t}(0,0,0)$ at level of significance 0.1 . Table 7 shows the maximum likelihood estimation for the selected final model with input variables of GDP and consumer price index and an output variable of defense expenditure.

After deciding the parameters of the Transfer Function model, it is necessary to estimate the model for noise series of the Transfer Function model based on the residual series of the model. Table 8 shows estimates and standard errors of parameter from the final Transfer Function model for noise series. In Table 8, the $p$-value of Portmanteau's chi-square test for verification of autocorrelation of residual series is satisfactory that the null hypothesis, "There is no correlation", cannot be rejected, which shows this residual series is a random series such as white noise. Hence, the final Transfer Function model is a good fitted model.

The cross-correlations of the final residuals and prewhitened input time series in Table 9 and Table 10 suggest that both series are independent. The hypothesis "The cross-correlation is equal to 0 " cannot be rejected since the $p$-values of both series are adequate.

From Table 7, Transfer Function model is derived as follows:

$$
\nabla \sqrt{z}_{t}=\frac{w_{s 1}(B)}{\delta_{r 1}(B)} B^{b 1} \nabla \sqrt[3]{g_{t}}+\frac{w_{s 2}(B)}{\delta_{r 2}(B)} B^{b 2} \nabla \sqrt{x_{t}}+\epsilon_{t},
$$


Table 9: Cross-correlation Check of Residuals with input $\sqrt[3]{g_{t}}$

\begin{tabular}{|c|c|c|c|c|c|c|c|c|c|}
\hline To Lag & $\chi^{2}$ & DF & $P$ & \multicolumn{5}{|c|}{ Autocorrelations } & \\
\hline 5 & 2.46 & 5 & 0.782 & 0.016 & 0.192 & -0.037 & -0.121 & 0.092 & -0.007 \\
\hline 11 & 11.83 & 11 & 0.376 & -0.196 & -0.301 & -0.171 & -0.146 & -0.230 & 0.038 \\
\hline 17 & 14.30 & 17 & 0.646 & 0.142 & 0.137 & 0.028 & 0.061 & 0.010 & 0.135 \\
\hline 23 & 17.81 & 23 & 0.768 & 0.066 & -0.173 & 0.007 & -0.124 & -0.117 & -0.157 \\
\hline
\end{tabular}

Table 10: Cross-correlation Check of Residuals with input $x_{t}$

\begin{tabular}{|c|c|c|c|c|c|c|c|c|c|}
\hline To Lag & $\chi^{2}$ & $\mathrm{DF}$ & $P$ & & & ocorrelat & & & \\
\hline 5 & 5.10 & 5 & 0.40 & 0.013 & 0.220 & 0.028 & -0.208 & -0.170 & -0.052 \\
\hline 11 & 9.39 & 11 & 0.59 & 0.076 & 0.055 & -0.035 & -0.026 & -0.272 & 0.142 \\
\hline 17 & 12.29 & 17 & 0.78 & -0.026 & -0.047 & 0.046 & 0.082 & 0.216 & 0.111 \\
\hline 23 & 16.79 & 23 & 0.82 & -0.003 & 0.025 & -0.144 & -0.062 & -0.164 & -0.240 \\
\hline
\end{tabular}

where $b 1=1, s 1=0, r 1=0, b 2=0, s 2=0$ and $r 2=0$.

Finally, the following model can be constructed using the estimates in Table 7.

$$
\sqrt{z}_{t}=\sqrt{z}_{t-1}+2.34\left(\sqrt[3]{g_{t-1}}-\sqrt[3]{g_{t-2}}\right)+2.54\left(\sqrt{x}_{t}-\sqrt{x_{t-1}}\right)+\epsilon_{t}
$$

As a result, defense expenditure can be estimated from the above formula.

\section{Forecasting}

After fitting a model, we estimate a future value at time $n$ based on the fitted model, while the actual value is unknown. We proposed the forecasts for the defense expenditure constructed by the ARIMA model and the Transfer Function model. We let the forecasted value $w_{n}(l)$ of $\sqrt{z_{t}}$ at time $t=n+l$. For the ARIMA model, we use the following equation to calculate the values of the forecast:

$$
w_{n}(l)=\mu+\phi_{1}^{l}\left(\sqrt{z_{n}}-\mu\right) .
$$

From the equation (2.1) of the fitted ARIMA model, $\hat{\mu}=17.82, \hat{\phi}_{1}=0.38$ and $\hat{\sigma}^{2}=74.08$. We can also obtain the standard error from the equation $\sqrt{\hat{\sigma}^{2}\left[1+\left(\hat{\phi}_{1}-\hat{\theta}_{1}\right)^{2} /\left(1-\hat{\phi}_{1}^{2}\right)\right]}$. The forecasts can be obtained by iterating recursion for equation (3.2) of the fitted transfer function model. The final forecasts of defense expenditures are obtained by multiplying the forecasted defense expenditure by the population of Korea. Table 11 shows the forecasts and standard errors of defense expenditures up to 2025. Based on our final fitted models and Korea's projected population from the National Statistical Office, we can predict that defense expenditures in 2025 will reach 48667.334 billion KRW.

AIC, SBC and MSE are model selection criteria and the best model is the model that minimizes that criterion. Table 12 shows that the Transfer Function model is an appropriate model for the defense expenditure since AIC, SBC and MSE values achieved in the Transfer Function model are smaller than those achieved in the ARIMA model.

\section{Concluding Remarks}

This paper proposed forecasting time series models for Korean defense expenditures using an ARIMA model based on past defense expenditures and a Transfer Function model with GDP, exchange rate and consumer price index as input time series. We omitted some complex formulas and intermediate results for the sake of simplicity. We can see that the Transfer Function model is an appropriate model for defense expenditures, since AIC, SBC and MSE values achieved in the Transfer Function model 
Table 11: The actual value and forecasts of the defense expenditure (Unit is one hundred - million KRW and standard error is given in parenthesis.)

\begin{tabular}{|c|c|c|c|c|c|}
\hline Year & Actual value & Arima model & residual & TF model & Residual \\
\hline 1972 & 1738 & $2048.07(24.82)$ & -310.07 & $1692.62(23.76)$ & -45.38 \\
\hline 1973 & 1843 & $2525.81(25.26)$ & -682.81 & $2080.82(24.18)$ & 237.82 \\
\hline 1974 & 2910 & $2510.10(25.70)$ & 399.90 & $2520.41(24.60)$ & -389.59 \\
\hline 1975 & 4588 & $4230.71(26.14)$ & 357.29 & $4144.26(25.02)$ & -443.74 \\
\hline 1976 & 7327 & $6402.23(26.56)$ & 924.77 & $5913.67(25.42)$ & -1413.33 \\
\hline 1977 & 9626 & 9919.77 (26.97) & -293.77 & $9154.22(25.82)$ & -471.78 \\
\hline 1978 & 12223 & $12126.29(27.39)$ & 96.71 & $11759.77(26.22)$ & -463.23 \\
\hline 1979 & 15366 & $15043.59(27.80)$ & 322.41 & $15497.76(26.62)$ & 131.76 \\
\hline 1980 & 22465 & $18651.38(28.24)$ & 3813.62 & $19976.29(27.03)$ & -2488.71 \\
\hline 1981 & 26979 & $28073.30(28.69)$ & -1094.30 & $27583.40(27.46)$ & 604.40 \\
\hline 1982 & 31207 & $31513.18(29.13)$ & -306.18 & $31508.74(27.89)$ & 301.74 \\
\hline 1983 & 32741 & $35797.82(29.57)$ & -3056.82 & $34314.00(28.30)$ & 1573.00 \\
\hline 1984 & 33061 & $36173.76(29.93)$ & -3112.76 & $36142.07(28.65)$ & 3081.07 \\
\hline 1985 & 36892 & $35964.39(30.23)$ & 927.61 & $36168.02(28.94)$ & -723.98 \\
\hline 1986 & 41580 & $41488.20(30.53)$ & 91.80 & $39937.96(29.23)$ & -1642.04 \\
\hline 1987 & 47454 & $46742.86(30.83)$ & 711.14 & $45951.69(29.52)$ & -1502.31 \\
\hline 1988 & 55202 & $53367.08(31.14)$ & 1834.92 & $53643.52(29.81)$ & -1558.48 \\
\hline 1989 & 60148 & $62231.10(31.45)$ & -2803.10 & $62228.33(30.10)$ & 2080.33 \\
\hline 1990 & 66378 & $66139.10(31.76)$ & 238.90 & $67276.22(30.40)$ & 898.22 \\
\hline 1991 & 74764 & 73146.07 (32.07) & 1617.93 & $76980.87(30.70)$ & 2216.87 \\
\hline 1992 & 84100 & $82782.40(32.41)$ & 1317.60 & $85548.42(31.02)$ & 1448.42 \\
\hline 1993 & 92154 & $92794.77(32.74)$ & -640.77 & $92959.59(31.34)$ & 805.59 \\
\hline 1994 & 100753 & 100554.09 (33.07) & 198.91 & $102370.98(31.66)$ & 1617.98 \\
\hline 1995 & 110743 & $109656.02(33.40)$ & 1086.98 & $112668.48(31.98)$ & 1925.48 \\
\hline 1996 & 122434 & $120470.78(33.72)$ & 1963.22 & $124152.68(32.28)$ & 1718.68 \\
\hline 1997 & 137865 & $133216.20(34.04)$ & 4648.80 & $134356.78(32.59)$ & -3508.22 \\
\hline 1998 & 138000 & $150333.41(34.29)$ & -12333.41 & $151807.33(32.82)$ & 13807.33 \\
\hline 1999 & 137490 & $144287.13(34.53)$ & -6797.13 & $139016.41(33.06)$ & 1526.41 \\
\hline 2000 & 144774 & $143715.94(34.82)$ & 1058.06 & $147187.43(33.34)$ & 2413.43 \\
\hline 2001 & 153884 & $154131.10(35.08)$ & -247.10 & $156936.89(33.58)$ & 3052.89 \\
\hline 2002 & 163640 & $163983.21(35.28)$ & -343.21 & $163765.12(33.77)$ & 125.12 \\
\hline 2003 & 175148 & $174237.35(35.45)$ & 910.65 & $176974.49(33.94)$ & 1826.49 \\
\hline 2004 & 189412 & $186534.32(35.59)$ & 2877.68 & 185772.85 (34.07) & -3639.15 \\
\hline 2005 & 211026 & 201960.83 (35.66) & 9065.17 & $200554.36(34.14)$ & -10471.64 \\
\hline 2006 & 225129 & $227775.31(35.83)$ & -2646.31 & 219874.85 (34.30) & -5254.15 \\
\hline 2007 & 244972 & $238751.04(36.00)$ & 6220.96 & $235275.59(34.46)$ & -9696.41 \\
\hline 2008 & 266490 & $262017.93(36.26)$ & 4472.07 & $262489.58(34.71)$ & -4000.42 \\
\hline 2009 & 289803 & $283728.73(36.43)$ & 6074.27 & $278945.66(34.88)$ & -10857.34 \\
\hline 2010 & 295627 & $308399.94(36.60)$ & -12772.94 & $301543.75(35.04)$ & 5916.75 \\
\hline 2011 & 314031 & 308114.35 (36.88) & 5916.65 & 319011.48 (35.30) & 4980.48 \\
\hline 2012 & 329576 & 330682.07 (37.04) & -1106.07 & $327455.38(35.46)$ & -2120.62 \\
\hline 2013 & 344970 & $345612.61(37.20)$ & -642.61 & $340648.36(40.66)$ & 4321.64 \\
\hline 2014 & . & $361148.67(37.35)$ & . & $354283.63(98.20)$ & . \\
\hline 2015 & . & $377851.78(109.35)$ & . & $367790.70(173.53)$ & . \\
\hline 2016 & . & 394992.40 (198.07) & . & 381177.15 (270.93) & . \\
\hline 2017 & . & $412522.43(294.06)$ & . & $394430.61(394.17)$ & . \\
\hline 2018 & . & $430406.65(393.28)$ & . & $407529.58(546.61)$ & . \\
\hline 2019 & . & $448629.59(494.04)$ & . & $420463.80(731.17)$ & . \\
\hline 2020 & . & $467180.06(595.66)$ & . & $433224.67(950.45)$ & . \\
\hline 2021 & . & $486051.62(697.85)$ & . & 445807.56 (1206.69) & . \\
\hline 2022 & . & $505226.63(800.42)$ & . & 458197.93 (1501.83) & . \\
\hline 2023 & . & $524677.65(903.24)$ & . & $470373.46(1837.47)$ & . \\
\hline 2024 & . & $544436.62(1006.31)$ & . & $482365.88(2215.18)$ & . \\
\hline 2025 & . & $564469.42(1109.51)$ & . & $494148.06(2636.07)$ & . \\
\hline
\end{tabular}


Table 12: AIC, SBC and MSE for ARIMA and Transfer Function model.

\begin{tabular}{cccc}
\hline \hline Model & AIC & SBC & MSE \\
\hline ARIMA & 309.26 & 312.78 & 74.78 \\
Transfer function & 293.02 & 296.45 & 70.91 \\
\hline \hline
\end{tabular}

are smaller than those achieved in the ARIMA model. The forecasting accuracy improved with the Transfer Function model. We verified that the proposed forecasting models are mathematically feasible to help provide basic data to estimate defense expenditures. We would like to also compare the trend of national defense expenditures on the two Koreas, but we cannot obtain proper data. GARCH models account for certain characteristics such as Volatility that are commonly associated with financial time series; therefore, it is not considered an alternative model. In the next study, we will analyze national defense expenditures compared to the GDP of Korea and neighboring nations (China, Russia and Japan) and will consider other time series models such GARCH models.

\section{References}

Aizenman, J. and Glick, R. (2006). Military expenditure, threats, and growth, Journal of Trade and Economic Development, 15, 129-155.

Anderson, T. W. (1971). The Statistical Analysis of Time Series, John Wiley \& Sons, Inc.

Baek, J. O., Sung, C. G. and Park, J. H. (2002). The analysis on Economic roles of national defense sector, The Quarterly Journal of Defense Policy Studies, 57, 65-90.

Box, G. E. P. and Jenkins, G. M. (1976). Time Series Analysis : Forecasting and Control, 2nd ed., Holdon-Day, San Francisco.

Chan, S. and Mintz, A. (2002). Defense, Welfare, and Growth, Routledge.

Korean Defense Ministry (2006). National Defense Reform 2020 and National Defense Expenditure, Ministry of National Defense.

Mintz, A. and Huang, C. (1990). Defense expenditures, economic growth, and the peace dividend, The American Political Science Review, 84, 1283-1293.

Rothschild, K. W. (1973). Military expenditure, exports and growth, Kyklos, 26, 804-814.

William, W. S. W. (1996). Time Series Analysis Univariate and Multivariate Methods, AddisonWesley Publishing Company. 